\title{
Patient Specific 3D Image-Based Radiation Dose Estimates for 90Y Microsphere Hepatic Radioembolization in Metastatic Tumors
}

\section{Andrew Kennedy ${ }^{1,2 *}$, William Dezarn ${ }^{3}$ and Alec Weiss ${ }^{3}$}

${ }^{1}$ Co-Medical Director, Wake Radiology Oncology, 300 Ashville Ave., Suite 110, Cary, NC, 27518 USA

${ }^{2}$ Adjunct Associate Professor, Department of Biomedical Engineering, Department of Mechanical and Aerospace Engineering, North Carolina State University, Raleigh, NC, USA

${ }^{3}$ Department of Biologic Systems Engineering, Campus Box 7910, Broughton Hall 4160, North Carolina State University, Raleigh, NC 27695-7910 USA

\begin{abstract}
Introduction: Hepatic brachytherapy using either resin or glass ${ }^{90} \mathrm{Y}$ microspheres is an established therapy for unresectable primary and metastatic tumors. Unlike conventional brachytherapy, microsphere brachytherapy has no software currently available for pretreatment evaluation and radiation planning. A non-MIRD radiation dose calculation approach is desired to accurately utilize spatial relationships in the liver and tumor distribution.

Materials and methods: A newly developed software tool employing the technetium-99m macro aggregated albumin (99mTc-MAA) SPECT 3-D dataset and CT scan was used to estimate the likely absorbed dose in normal liver and tumor tissue from ${ }^{90} \mathrm{Y}$ microsphere brachytherapy (radioembolization). Monte Carlo algorithms were utilized to maximize true 3D dose estimates for each patient's unique liver and tumor geometry. Clinical correlation was completed regarding toxicity, imaging response, and complications as an independent measure of the software's usefulness in predicting radiation effects. Comparisons were made to MIRD, Body Surface Area method, and physician prescription for ${ }^{90} \mathrm{Y}$ activity.

Results: The software performed accurately in estimating absorbed dose in phantom testing. Patient data from 50 consecutive patients with metastatic tumors (26 colon, 24 neuroendocrine) to the liver receiving 59 radioembolization treatments were studied. The software estimate of median normal liver and tumor absorbed doses were 27.6 Gy and 41.2 Gy, respectively.

Conclusions: The use of pretreatment ${ }^{99 m}$ TC-MAA SPECT co-registered to a CT scan provides useful and unique data for a newly developed non-MIRD, Monte Carlo-based radiation dosimetry software program in ${ }^{90} \mathrm{Y}$ microsphere brachytherapy. Software estimates of radiation dose preserving critical spatial information in the liver and tumors appeared reasonable based on clinical outcomes. Further testing and refinement of the software interface is ongoing with plans to distribute it to research organizations.
\end{abstract}

Keywords: Yttrium-90; Dose volume histograms; Dosimetry; SIRT; Brachytherapy

\begin{abstract}
Abbreviations: RE: Radioembolization; MIRD: Medical Internal Radiation Dose; BSA: Body Surface Area; ${ }^{99 \mathrm{~m} T c-M A A: ~}{ }^{99 \mathrm{~m}} \mathrm{Tc}$ Macro Aggregated Albumin; SPECT: Single Photon Emission Computed Tomography; NET: Neuroendocrine Tumor; ROI: Region of Interest; RILD: Radiation Induced Liver Disease; REILD: Radioembolization Induced Liver Disease; CEA: Carcinoembryonic Antigen; TLG: Total Lesion Glycolysis; TOF: Time of Flight
\end{abstract}

\section{Introduction}

Radioembolization (RE) is defined as the intra-arterial delivery of micron-sized microspheres embedded with a radioisotope (usually Yttrium-90, ${ }^{90} \mathrm{Y}$ ) that become permanently embedded in a tumor preferentially to normal tissues. The technique is accomplished through fluoroscopic guidance of intravascular catheters with endpoints of adequate coverage of tumor by implanted microspheres inferred by changes in tumor vascular flow [1,2]. Patient selection requires liverdominant tumor burden, adequate liver function and arterial access to the liver as a minimum [3]. Despite the wealth of published literature on technical aspects of successful RE [4-8] over the past 40 years of its existence, there have been few investigations on optimizing radiation activity selection and actual dosimetry [1,4,9-33]. One reason for this is that there is no software solution for estimating radiation dose delivery in $\mathrm{RE}$ as there is with conventional brachytherapy (interstitial and intracavitary). Guidance for dose selection has recently been updated by an expert panel for both resin and glass microspheres used in RE [34].
Estimating the proper activity of ${ }^{90} \mathrm{Y}$ microspheres to implant in a given patient is not an exact exercise. There are two types of radioactive microspheres commercially available, and both use ${ }^{90} \mathrm{Y}$ and are approximately the same diameter; but the glass spheres have a larger specific gravity $(3.6 \mathrm{gm} / \mathrm{dL})$ compared with resin spheres $(1.6$ $\mathrm{gm} / \mathrm{dL}$ ). This enables the glass spheres to carry fifty times more activity than resin spheres $(2500 \mathrm{~Bq}$ vs. $50 \mathrm{~Bq})$, and thus a typical treatment only requires up to 8 million glass spheres whereas the resin sphere treatment employs upwards of 40 million spheres [22,25].

The method of choosing an activity of ${ }^{90} \mathrm{Y}$ in $\mathrm{RE}$ is dependent upon which of the two ${ }^{90} \mathrm{Y}$ microsphere types is being used. The recommended method for glass microspheres is via the schema developed by the Medical Internal Radiation Dose (MIRD) Committee of the Society of Nuclear Medicine. It assumes uniform distribution of isotope throughout the liver [35]. Although it is known that this is clearly

*Corresponding author: Andrew Kennedy, MD, FACRO, Wake Radiology Oncology, 300 Ashville Ave., Suite 110, Cary, North Carolina 27518, Tel: (919) 8544588; Fax: (919) 854-9950; E-mail: akennedy@wakerad.com

Received April 26, 2011; Accepted May 26, 2011; Published June 15, 2011

Citation: Kennedy A, Dezarn W, Weiss A (2011) Patient Specific 3D Image-Based Radiation Dose Estimates for 90Y Microsphere Hepatic Radioembolization in Metastatic Tumors. J Nucl Med Radiat Ther 2:111. doi:10.4172/2155-9619.1000111

Copyright: (C) 2011 Kennedy A, et al. This is an open-access article distributed under the terms of the Creative Commons Attribution License, which permits unrestricted use, distribution, and reproduction in any medium, provided the original author and source are credited. 
not the case in RE [10-12,15,25], the approach has been validated in prospective clinical trials and in thousands of patients treated over the past two decades. The "dose" in Gy suggested by the MIRD equations for $\mathrm{RE}$ is considered a close approximation if the tumor is a discrete region of interest (ROI) and the liver and lungs can also be clearly identified [32]. A similar approach for resin microspheres, termed the "partition method," is also considered reliable and valid, but only in the special circumstance as noted with a discrete ROI, which is seldom present in metastatic tumors in the liver [20,21]. Thus the most commonly used method for resin microsphere radioactivity selection is the Body Surface Area (BSA) approach and historically, the Empiric approach, but currently the latter is not commonly used [25]. With resin microspheres, the BSA method considers the mass of tumor and normal liver, and reduces activity to be delivered by a constant plus the patient's BSA. The BSA and Empiric resin activity calculations have been used in prospective clinical trials and are considered valid [36].

We have developed a software tool for the estimation of radiation dose absorption based on the voxel value of nuclear medicine imaging via Monte Carlo kernels. Among the 11 isotopes validated in this software module are yttrium-90 $\left({ }^{90} \mathrm{Y}\right)$ and technetium-99 $\left({ }^{99 \mathrm{~m}} \mathrm{Tc}\right)$. Because all patients worldwide must first undergo a hepatic angiogram and ${ }^{99 \mathrm{~m}} \mathrm{Tc}$ macro aggregated albumin ( $\left.{ }^{99 \mathrm{~m}} \mathrm{Tc}-\mathrm{MAA}\right)$ scan prior to RE, we decided this was potentially the best point at which to begin a radiation preplanning software approach. There are conflicting reports regarding the usefulness and clinical applicability of ${ }^{99 \mathrm{~m} T c-M A A}$ in predicting the distribution of radioactive microspheres in hepatic RE $[13,14,19]$. The utility of ${ }^{99 m}$ Tc-MAA single photon emission computed tomography (SPECT) in dose planning has yet to be determined and thus we undertook a study of a pretreatment software estimation of radiation dose absorption based on a simulated treatment with an abdominal CT scan fused to a ${ }^{99 \mathrm{~m}} \mathrm{Tc}-\mathrm{MAA}$ SPECT dataset. The resolution of ${ }^{99 \mathrm{~m}} \mathrm{Tc}-$ MAA SPECT is at best $2 \mathrm{~cm}$ and thus the distribution of activity will be an average of the area that is at least $2 \mathrm{~cm}^{2}$ and may include a larger area. Kennedy and others have shown microdosimetry 3D plots of $\mathrm{RE}$ in human patients. The distribution of radiation was extremely heterogeneous, as is expected in brachytherapy, with ranges of $25 \mathrm{~Gy}$ to $3,000 \mathrm{~Gy}$ over a $4 \mathrm{~cm}$ area $[12,15,25]$.

\section{Materials and Methods}

\section{Software structure}

Dose calculation was accomplished via a research build using a fast Hartley transform [37,38] to calculate the convolution integral. The dose distribution of a given activity distribution can be calculated by convolving the activity distribution with a Monte Carlo calculated dose kernel. For a homogeneous medium, the dose calculation is conducted using the convolution integral:

$$
D(\vec{r})=\int A\left(\vec{r}^{\prime}\right) K\left(\vec{r}-\vec{r}^{\prime}\right) d \vec{r}^{\prime}
$$

Where:

$\mathrm{D}(\overrightarrow{\mathrm{r}})$ is the absorbed dose (Gy) in the voxel centered at location $\overrightarrow{\mathrm{r}}$

$\mathrm{A}\left(\overrightarrow{\mathrm{r}}^{\prime}\right)$ is the cumulated activity (Bq-s) at location $\overrightarrow{\mathrm{r}}$

$\mathrm{K}\left(\overrightarrow{\mathrm{r}}-\overrightarrow{\mathrm{r}}^{\prime}\right)$ is the spatially invariant dose deposition kernel (Gy Bq $\mathrm{B}^{-1} \mathrm{~s}^{-1}$ between location $\vec{r}$ and source location $\vec{r}$

The dose kernels used in this work were calculated by the authors using the EGSnrc Monte Carlo system [39]. The energy spectrum from ICRU72 [40] for the beta decay of Yttrium $90\left({ }^{90} \mathrm{Y}\right)$ was entered into EGSnrc. Cubic dose kernel voxels were calculated for 16 voxel sizes ranging from 2 to $6 \mathrm{~mm}$. Linear interpolation was then used to calculate the dose kernel corresponding to the given activity distribution voxel size.

The developers of PLanUNC (PLUNC), 1 a radiation treatmentplanning software system, granted us permission to adapt it as needed for research requiring the manipulation of $\mathrm{CT}$ and other $3 \mathrm{D}$ image datasets (DICOM). The platform was also used to calculate the patientspecific tumor and normal liver dose volume histograms. The threedimensional dose distributions were visualized within the PLUNC system either alone or superimposed on a CT image set that has been registered to the activity distribution.

\section{Phantom validation of dose calculation}

An internal software test of the radiation dose calculation function was carried out prior to clinical data analysis. This test was accomplished with the given specification that when $1 \mathrm{GBq}$ of ${ }^{90} \mathrm{Y}$ is uniformly distributed in a phantom having a mass of $1 \mathrm{~kg}$, a total dose of $49.8 \mathrm{~Gy}$ is expected to be delivered [32]. Taking this as a benchmark, two $10 \times 10 \times 10 \mathrm{~cm}^{3}$ test phantoms of $1 \mathrm{~kg}$ mass were created within the PLUNC system, one with $2.5 \mathrm{~mm}$ and another with $5 \mathrm{~mm}$ voxel sizes. The results from these tests were consistent and therefore we felt comfortable applying the software in the clinical setting of previously treated patients.

If $1 \mathrm{GBq}$ of ${ }^{90} \mathrm{Y}$ is uniformly distributed in a phantom with a mass of $1 \mathrm{~kg}$, the MIRD dosimetry system calculates a total dose of $49.8 \mathrm{~Gy}$ delivered to the mass [32]. In equation form, the MIRD dosimetry for ${ }^{90} \mathrm{Y}$ is given as

$$
\mathrm{D}[\mathrm{Gy}]=\frac{49.8 \mathrm{~A}[\mathrm{GBq}]}{\mathrm{m}[\mathrm{kg}]}=\frac{49800 \mathrm{~A}[\mathrm{GBq}]}{\mathrm{s}^{3}[\mathrm{~cm}]}
$$

Where the density of water is accepted as $1 \mathrm{~g}$ or equal to a mass of $1 \mathrm{~cm}^{-3}$; and $\mathbf{s}$ is the side of the cube containing the activity. The MIRD equation is taken as the accepted benchmark dosimetry system for this work. Two $10 \times 10 \times 10 \mathrm{~cm}^{3}$ test phantoms with water density were created within the PLUNC system, one with $2.5 \mathrm{~mm}$ and another with $5 \mathrm{~mm}$ voxel sizes. Table 1 details the agreement of the MIRD equation dose to the dose convolution calculation value in the center of the phantom to minimize edge effects.

An additional test on the algorithm was conducted with three $8 \times 8 \times 8 \mathrm{~cm}^{3}$ test phantoms created within the PLUNC system with water density and varying activity. Five-millimeter voxel sizes were used for this comparison. Table 2 details the numerical results and Figure 1 displays the isodose lines for this case. The numerical results demonstrate that the convolution algorithm implemented in this work concurs with accepted dosimetry for a non-trivial case. The graphical isodose display demonstrates the correct display of isodose lines for a non-uniform case. Both results support the conclusion that the algorithm is functioning correctly at the voxel level.

\section{Radiation source in radioembolization}

Yttrium-90 is a beta-emitter that decays to stable zirconium-90, with a half-life of 64.2 hours and an average energy of $0.94 \mathrm{MeV}$, yielding tissue penetration of $2.5 \mathrm{~mm}$ and a maximum range of $1.1 \mathrm{~cm}$. Two radioactive microsphere products are available in North America, Asia, and Europe: 1) a glass sphere (TheraSphere ${ }^{\circledR}$, MDS Nordion, Inc., Ontario, Canada), with a diameter of $25 \mu \mathrm{m}$; and 2) a resin sphere (SIRSpheres $^{\circledR}$, Sirtex Medical Limited, Sydney, Australia), with a diameter of $32 \mu \mathrm{m}$. Both microspheres permanently embed ${ }^{90} \mathrm{Y}$ within their structure, and no clinically significant amount of ${ }^{90} \mathrm{Y}$ escapes from the 
microspheres when in the patient [25]. For this report, all patients had received only resin microspheres; however the MIRD method of dose calculation for glass microsphere treatments was applied retrospectively as a comparator.

\section{Patients}

The charts of 50 consecutive patients with metastatic hepatic tumors who underwent resin microsphere RE were retrospectively studied for the following: imaging response by Response Evaluation Criteria In Solid Tumors (RECIST), post treatment side effects per the National Cancer Institute Common Terminology Criteria for Adverse Events v3.0 (NCI CTCae 3.0), and details of radiation dose planning and delivery. These included normal liver volume, tumor volume, and lung shunt fraction, BSA activity estimates; physician prescribed activity, and delivered activity. In addition, the MIRD-estimated absorbed dose (Gy) that is utilized in glass microsphere RE treatments was calculated for all. This type of dose estimate in resin ${ }^{90} \mathrm{Y}$ microsphere RE has not been done before but is recommended in an upcoming guidance report to facilitate comparison among papers [41]. Every patient underwent a full history and physical, liver function tests, and CT scan at 6 and 12 weeks post RE and every 3 months thereafter. Toxicity due to liver radiation therapy was recorded according to CTCae3.02 and was focused on three areas: 1) gastrointestinal (nausea, emesis, pain); 2) constitutional (weight loss, fatigue, fever); and 3) biochemical (alkaline phosphatase, total bilirubin, AST, ALT). Radiographic response was measured by CT or magnetic resonance imaging (MRI) at 12 weeks post radiation according to RECIST criteria.

\section{Imaging source data}

The original DICOM files obtained were of pretreatment evaluations that included CT scan of the abdomen and ${ }^{99 \mathrm{~m}} \mathrm{Tc}-\mathrm{MAA}$ SPECT scan used in determining patient eligibility for RE and in activity calculations. Although MRI and positron emission tomography (PET)

\begin{tabular}{|l|l|l|l|}
\hline $\begin{array}{l}\text { Voxel Size } \\
(\mathbf{m m})\end{array}$ & $\begin{array}{l}\text { Expected Dose } \\
(\text { Gy) }\end{array}$ & $\begin{array}{l}\text { Calculated Dose } \\
(\text { Gy) }\end{array}$ & Percent Difference \\
\hline 2.5 & 49.8 & 51.1 & $+2.6 \%$ \\
\hline 5 & 49.8 & 50.6 & $+1.6 \%$ \\
\hline
\end{tabular}

Table 1: Comparison of calculated phantom dose to expected dose.

\begin{tabular}{|l|l|l|l|}
\hline $\begin{array}{l}\text { Activity } \\
\text { (GBq) }\end{array}$ & MIRD Dose (Gy) & Convolution Dose (Gy) & Percent Difference \\
\hline 0.7 & 68.1 & 69.2 & $+1.6 \%$ \\
\hline 0.2 & 19.5 & 19.8 & $+1.7 \%$ \\
\hline 0.1 & 9.73 & 9.9 & $+1.5 \%$ \\
\hline
\end{tabular}

Table 2: Comparison of calculated phantom dose to expected dose varying activity.

\begin{tabular}{|l|l|l|}
\hline Gender & N & $\%$ \\
\hline Male & 26 & $52 \%$ \\
\hline Female & 24 & $48 \%$ \\
\hline Total & 50 & $100 \%$ \\
\hline Tumor Histology & $\mathbf{N}$ & $\%$ \\
\hline Colorectal & 24 & $48 \%$ \\
\hline Neuroendocrine & 26 & $52 \%$ \\
\hline Total & 50 & $100 \%$ \\
\hline Treatment Volume/Target & $\mathbf{N}$ & $\%$ \\
\hline Whole Liver & 44 & $88 \%$ \\
\hline Right Lobe & 4 & $8 \%$ \\
\hline Left Lobe & 2 & $4 \%$ \\
\hline Total & 50 & $100 \%$ \\
\hline
\end{tabular}

Table 3: Patient and Tumor Characteristics. scans could have been used in the software module, we limited input to only CT for fusion with ${ }^{99 \mathrm{~m}} \mathrm{Tc}-\mathrm{MAA}$ SPECT to reduce potential bias as not every patient had these studies. The CT slice thickness was always $5 \mathrm{~mm}$ and either a non-contrast or venous phase image set was used if available. The arterial phase images were reviewed for neuroendocrine tumors (NET) as they can show tumors more clearly in some patients, but mostly the venous phase was found to be adequate in pre planning.

\section{Statistics}

Significance between groups was analyzed by unpaired t test, two tailed, with significance level of $\mathrm{p}=0.05$ unless otherwise noted. If Gaussian distribution was not known, the Mann-Whitney, two-tailed test was implemented. ANOVA, two tailed, was used for multiple comparisons among factors and groups.

\section{Results}

\section{Phantom testing/validation}

Summary data in Tables 1 and 2 show the accuracy of the dose calculation to the expected dose value in the center of the phantom to minimize edge effects. These results demonstrate that the convolution method used in this work is accurate as illustrated in Figure 1.

\section{Software use}

The process of co-registering MAA and CT scans was completed manually based on the liver contour and boney landmarks. Early on in our experience we placed several fudicial markers filled with ${ }^{99 \mathrm{~m}} \mathrm{Tc}$ on the patient's skin, at approximately midline, left and right lateral positions at the level of the liver. Because artifact from the gamma emissions in the fudicials interfered with dose calculations, we moved the markers to a distance from the liver. However, these markers were not helpful in remote locations as the skin surface often was not accurate in registering the image sets. Most patients had bilobar disease and could be registered in a few minutes when the operator visually aligned the liver and tumor of the CT and MAA. Dose calculations were produced quickly once the fusion of image sets was completed.

\section{Patients}

A thorough analysis of the clinical parameters, patient specific factors, imaging response, and toxicity of hepatic radiotherapy was conducted. With these data we were able to evaluate from the clinical perspective whether the normal liver and tumor doses reported by the software were realistic and in line with expectations. It should be noted that in our experience of more than 300 glass microsphere treatments, the median activity delivered has been $4.45 \mathrm{GBq}$, which is expected to be considerably higher than any resin microsphere treatment protocol for reasons discussed earlier in our report.

The details of clinical and treatment data are summarized in Tables 3-4. The percentages of male and female patients differed between the colon and neuroendocrine cancer groups, which was anticipated from the known epidemiology of these malignancies in the US. Whole liver treatments were performed in all NET patients and in $78 \%$ of the colon patients. The lobar-treated colon patients had undergone prior partial hepatectomy and thus, while the whole remaining liver volume was treated, in reality, it was only a left- or right-lobe treatment. This may explain the significant difference in normal liver volume between NET and colon patients $(p=0.053)$. No difference existed between tumor volume of NET and colon ( $\mathrm{p}=0.33$ ) patients. The amount of lung shunting and the BSA calculations showed equivalence in both tumor types $(\mathrm{p}=0.734)$, Table 4 . 
Citation: Kennedy A, Dezarn W, Weiss A (2011) Patient Specific 3D Image-Based Radiation Dose Estimates for 90Y Microsphere Hepatic Radioembolization in Metastatic Tumors. J Nucl Med Radiat Ther 2:111. doi:10.4172/2155-9619.1000111

The combined dose calculations for NET and colon cohorts showed significant differences in the activity of ${ }^{90} \mathrm{Y}$ calculated to be delivered by the BSA method versus physician prescription $(\mathrm{p}<0.003)$ and the actual activity that was delivered to the patient $(\mathrm{p}<0.0001)$. Comparison of the physician prescription to delivered activity was also significant $(\mathrm{p}<0.017)$. No significant differences were found in comparisons of BSA suggested activities in NET versus in colon $(\mathrm{p}=0.544)$ despite the tumor volume differences.

Separate comparisons of the dose estimated by the software for tumor in NET and tumor in colon patients compared to the normal liver in NET and colon patients revealed a significant difference $(p=<0.0001)$ in favor of tumor receiving a greater dose. Similarly, the MIRD estimated doses were significantly higher in tumor than normal liver for both tumor types $(\mathrm{p}=<0.0001)$. Comparison of the dose estimate of the software with MIRD estimates in tumor were not significant in colon patients $(\mathrm{p}=0.16)$ but were significantly different $(\mathrm{p}=0.0003)$ in NET patients.

\begin{tabular}{|l|l|l|}
\hline Volumetrics & Median & Min-Max \\
\hline Normal Liver (cc) & 1893.8 & $945.7-3999.7$ \\
\hline Tumor (cc) & 196.6 & $4.9-2207.6$ \\
\hline Lung Shunt (\%) & 5.5 & $1.0-18.2$ \\
\hline BSA $\left(m^{2}\right)$ & 1.9 & $1.6-2.2$ \\
\hline${ }^{90}$ Y Activity Selection & Median & Min-Max \\
\hline BSA method (GBq) & 1.6 & $1.5-1.7$ \\
\hline MD Prescribed (GBq) & 1.5 & $1.5-1.8$ \\
\hline Delivered (GBq) & 1.4 & $1.4-1.9$ \\
\hline Dose Calculation & Median & Min-Max \\
\hline MIRD Normal Liver (Gy) & 49.9 & $44.6-55.2$ \\
\hline MIRD Tumor (Gy) & 49.9 & $44.6-55.2$ \\
\hline Software Normal Liver (Gy) & 27.6 & $22.8-32.4$ \\
\hline Software Tumor (Gy) & 41.2 & $35.7-46.8$ \\
\hline
\end{tabular}

Table 4: Physical Parameters and Radiation Calculations.

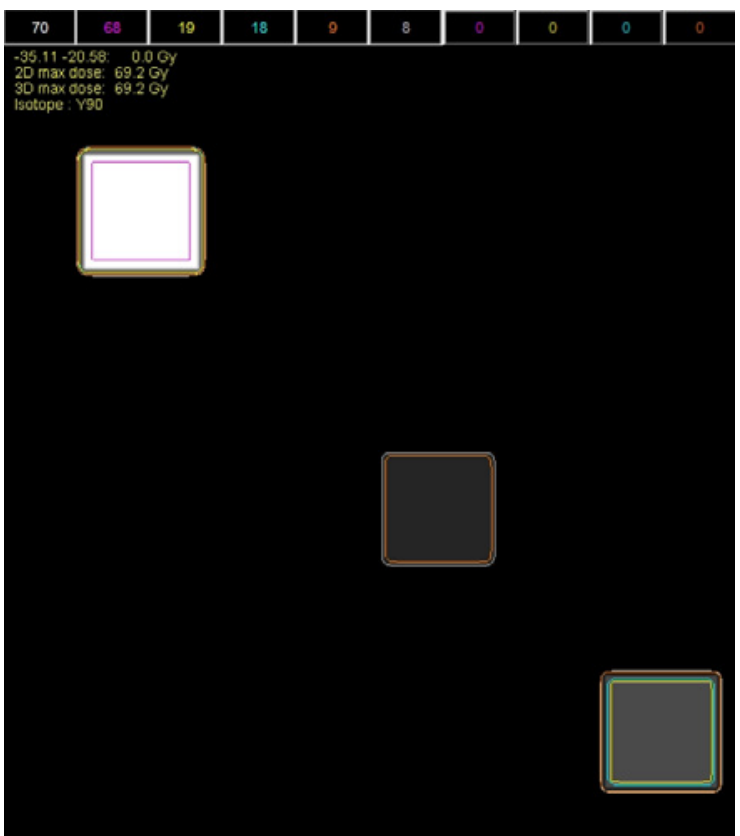

Figure 1: Screen shot of the testing set up of software dose calculation validation.

\section{Toxicity and response rate}

A total of 9 patients were found to have grade 1 or 2 CTCae3.0 toxicity. No patient experienced grade 3 or 4 and there were no grade 1 biochemical events. Three patients experienced gastrointestinal side effects: one grade 1 event each of nausea, emesis and abdominal pain. Among patients with remaining side effects, 3 patients developed grade 1 weight loss, 4 had grade 1 fever, and 4 had grade 1 fatigue. Two additional patients reported grade 2 fatigue symptoms. All patients completely recovered from all side effects by week 12 post treatment. There was no correlation between toxicity and radiation activity delivered, MIRD dose, or software dose estimates $(\mathrm{p}=\mathrm{ns})$.

RECIST response at 12 weeks post radiation in 50 patients was as follows: complete response in 3 patients (6\%), stable disease in $26(52 \%)$, partial response in $12(26 \%)$, and progressive disease in 7 (14\%). Since all patients were progressing at the time of treatment, we considered stable disease after treatment to represent a response for the purposes of analysis, and thus $44 / 50$ patients (88\%) were responders. Of the 7 patients that progressed after liver radiotherapy, 6 were colon cancer patients and only 1 patient had neuroendocrine carcinoma. Partial responders included $3 / 13$ patients with colon primaries and 10/13 with NET. There was no difference in the median dose to tumor as estimated by the software $(\mathrm{p}=0.244)$ for responders and non-responders.

\section{Discussion}

There have been a variety of attempts to describe the radiation dose delivered to tumors and normal liver from ${ }^{90} \mathrm{Y}$ microsphere therapy [9$21,24-26,28,30-33,42]$. It is noteworthy that despite the lack of accurate pre- and post-treatment dose estimates as are available in conventional brachytherapy, safety and efficacy are quite high in numerous histology types, especially colorectal, hepatocellular and neuroendocrine tumors [36]. However it is acknowledged that a major need in clinical progress for RE is development and validation of pre- and post-treatment dosimetry tools. Among the many advances to come with such tools, not the least will be the ability to compare between treatment arms in clinical studies the dose absorbed in liver and tumor, and the publication of dose-response data for metastatic and primary hepatic tumors.

The earliest attempts in dosimetry of ${ }^{90} \mathrm{Y}$ microsphere therapy were performed via intraoperative beta-radiation probes after Empiric activity selection $[9,10]$. Subsequently, most physicians adopted a semi Empiric approach using the tumor volume as a guide or utilized nuclear medicine formalism-MIRD [2,7,8,22,35]. Obvious disadvantages of both approaches abound, however through the years a range of carefully delivered activities of ${ }^{90} Y$ have been reported with very few complications of radiation overdose, known as radiation induced liver disease (RILD) $[16,24,25,27,43-46]$ or more recently as radioembolization induced liver disease (REILD) [29]. Reported fatalities from liver failure post $\mathrm{RE}$ have later been evaluated as resulting from excessive radiation for those particular patients. It is unknown whether this could have been avoided using dosimetry tools as opposed to the Empiric/MIRD approach that is presently used. Current activity selection for resin microspheres (Empiric, BSA) differs from glass microspheres (MIRD). There is no known association correlating BSA with liver volume, tumor volume or radiation sensitivity. The MIRD approach assumes uniform distribution of radiation throughout the liver and tumor, ignoring all spatial data to the contrary as heterogeneous deposition of microspheres is the key therapeutic advantage. Gulec applied MIRD and state of the art Monte Carlo methods to model ${ }^{90} \mathrm{Y}$ radiation dose deposition at the lobular level of normal liver [47]. 
Citation: Kennedy A, Dezarn W, Weiss A (2011) Patient Specific 3D Image-Based Radiation Dose Estimates for 90Y Microsphere Hepatic Radioembolization in Metastatic Tumors. J Nucl Med Radiat Ther 2:111. doi:10.4172/2155-9619.1000111

(A)

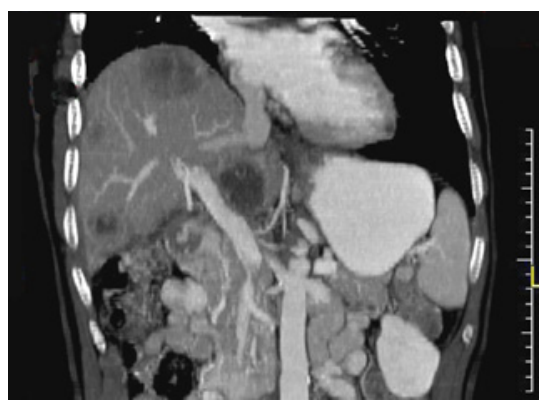

(C)

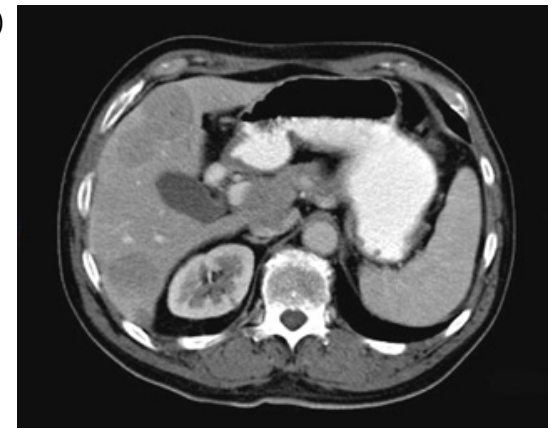

(E)

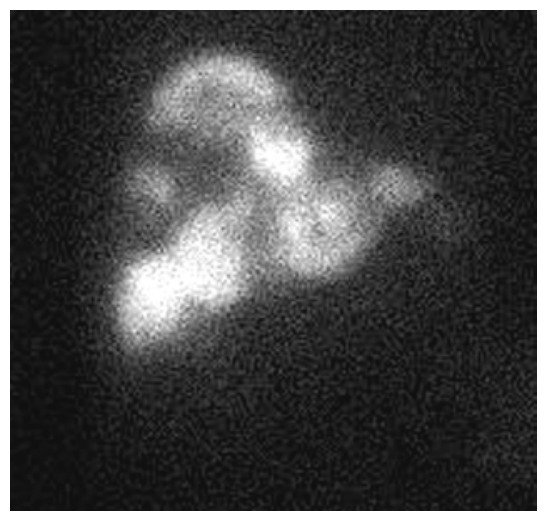

(G)

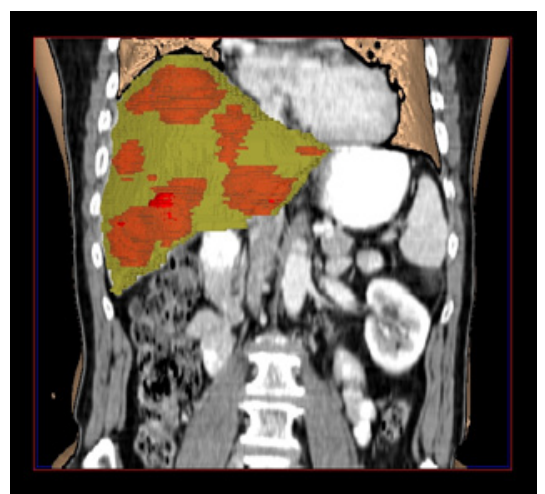

(B)

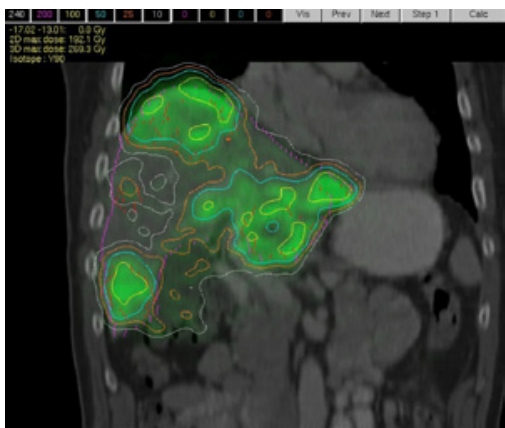

(D)

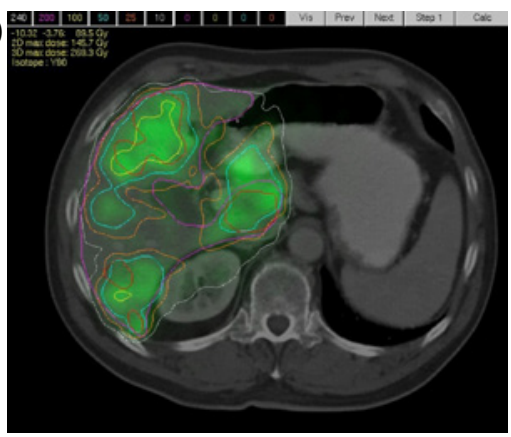

(F)

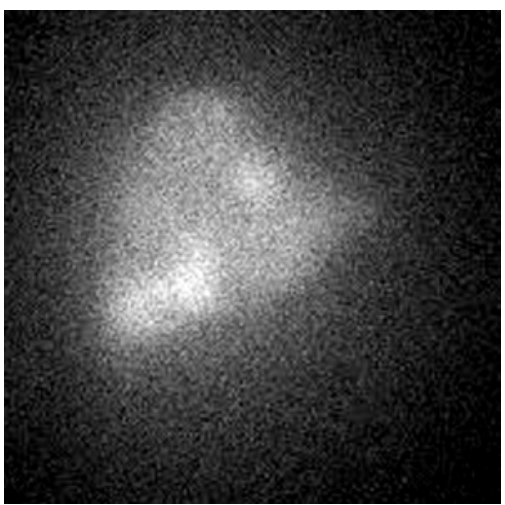

(H)

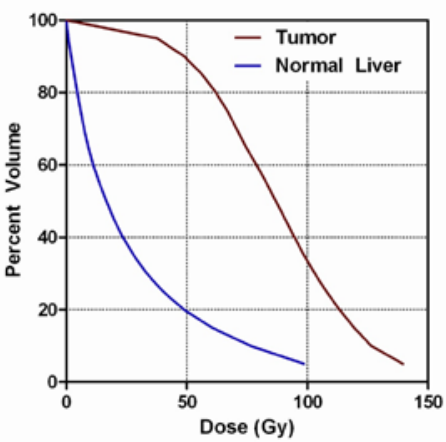

Figure 2: The following (A-H) are from a patient with neuroendocrine liver metastases who received whole liver ${ }^{90} \mathrm{Y}$ microsphere therapy. A. Coronal view of a diagnostic CT scan used for radiation treatment planning as the source of obtaining normal liver and tumor volume data. B. Software dose calculation screenshot in coronal view. outlined on each CT axial slice. Radiation cumulative doses are represented by isodose curves overlying the liver and a small amount of adjacent right kidney, duodenum and gastric antrum. C. Axial image from pretreatment diagnostic CT scan. D. Screenshot of axial view of dose calculation software showing mostly right hepatic lobe and a portion of the caudate lobe with isodose curves overlaid. E. Coronal view obtained by ${ }^{99 m}$ Tc-MAA SPECT scintigraphy highlighting the heterogeneous areas of increased albumin particle deposition in tumor versus normal liver. The tumor to normal ratio was greater than 9:1 via ROI measurements. F. Bremsstrahlung SPECT imaging obtained 30 minutes after implantation of ${ }^{90} \mathrm{Y}$ microspheres, coronal view. The count numbers and resolution are significantly less than the pretreatment MAA scintigraphy, however focal uptake in multiple tumors can be seen. G. Three-dimensional reconstruction of the pretreatment diagnostic CT scans in coronal view. This image was created by standard radiation treatment planning software for external beam therapy. This is the current method used by the authors to acquire volumetric data of normal liver and tumor for ${ }^{90} \mathrm{Y}$ microsphere activity planning. $\mathbf{H}$. Cumulative dose volume histogram of normal liver and tumor showing relative sparing of normal liver. 
Citation: Kennedy A, Dezarn W, Weiss A (2011) Patient Specific 3D Image-Based Radiation Dose Estimates for 90Y Microsphere Hepatic Radioembolization in Metastatic Tumors. J Nucl Med Radiat Ther 2:111. doi:10.4172/2155-9619.1000111

Page 6 of 8

(A)

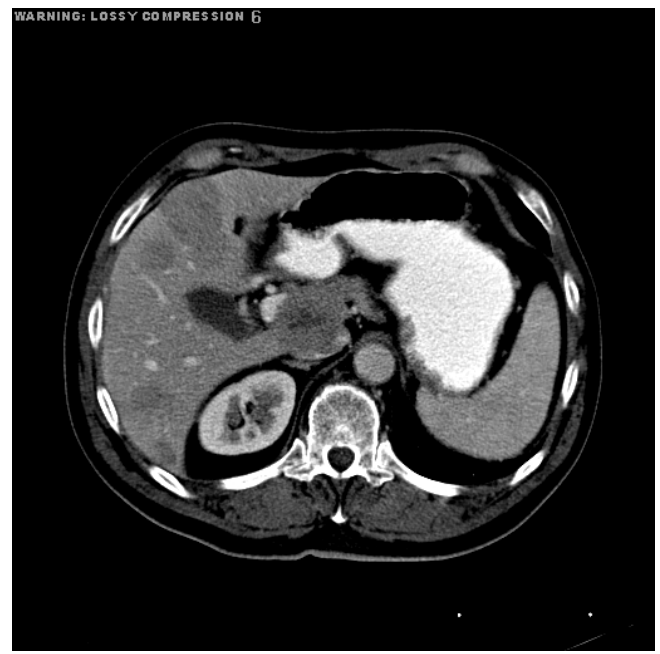

(B)

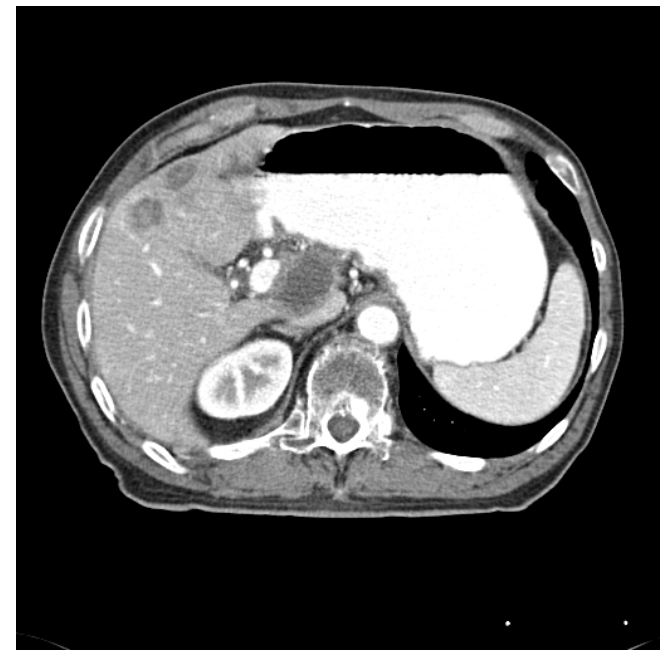

Figure 3: A. Pretreatment diagnostic CT axial image of the patient in Figure 2. B. Post-treatment diagnostic CT axial image of the same patient at the same portion of the liver at 9 months after ${ }^{90} \mathrm{Y}$ microsphere therapy, scored as a RECIST partial responder although no enhancement or lesion growth was ever seen in follow up. The software-estimated tumor dose was $86.6 \mathrm{~Gy}$ and normal liver was only $16.5 \mathrm{~Gy}$; MIRD estimate was $43.7 \mathrm{~Gy}$. The total activity delivered was $2.0 \mathrm{GBq}$, with tumor volume of $296.8 \mathrm{cc}$ and normal liver $2109.3 \mathrm{cc}$. This patient did not experience any grade 1-3 toxicities.

Dhabuwala et al. [13] studied the relationship of ${ }^{99 m}$ Tc-MAA scintigraphy and clinical outcomes at $4-6$ weeks post treatment, including response measures by $\mathrm{CT}$ and the carcinoembryonic antigen (CEA) tumor marker, in 58 metastatic colorectal cancer patients receiving resin microsphere RE. The authors concluded that the pattern of ${ }^{99 \mathrm{~m}} \mathrm{Tc}$-MAA uptake by colorectal liver tumors was not a predictor of tumor response after RE [13]. However there are important shortcomings in this report that may have affected their conclusions. The ${ }^{99 \mathrm{~m} T c-M A A}$ was planar, not SPECT, and therefore quite limited. Response by CT scan was not evaluated by RECIST or WHO criteria; rather they identified scans as reflecting disease reduction, stable disease, or progressive disease. The authors reported that a significant response was seen in at least $50 \%$ of the patients, regardless of whether they were judged to have a "hot" or "cold" tumor uptake pattern by ${ }^{99 m}$ Tc-MAA [13]. It is believed by most investigators that the maximum response by CT scan after RE occurs at approximately 12 weeks, and thus the early evaluation of CT and CEA could have been inaccurate. The same is true for CEA values after RE.

Flamen et al. [14] reported a study of 8 patients with colon cancer who received RE and were evaluated by comparison of ${ }^{99 \mathrm{~m}} \mathrm{Tc}-\mathrm{MAA}$ SPECT pre treatment and PET-CT post treatment using total lesion glycolysis (TLG) as the endpoint for response [14]. The liver voxel uptake values from ${ }^{99 \mathrm{~m}} \mathrm{Tc}$-MAA SPECT were converted to absolute ${ }^{90} \mathrm{Y}$ activity, and these values converted to a simulated absorbed dose (Gy) using simple MIRD formalism. Of note was the production of the first dose-response curve in liver brachytherapy. The authors noted a highly variable blood flow between lesions even in the same liver lobe and thus used individual tumor/normal liver ratios for each of the 39 lesions studied. This semi-quantitative MIRD method of dose estimation, correlated with fluorodeoxyglucose (FDG)-PET response, suggested there was a definite dose-response relationship in ${ }^{90} \mathrm{Y}$ RE for these patients. The mean absorbed dose in normal liver was $27 \mathrm{~Gy}(95 \% \mathrm{CI}$ : 22-33 Gy), with a responding tumor dose median of 46 Gy (22-110 Gy) and poorly responding tumor-lesion median dose of $20 \mathrm{~Gy}$ (1-68 Gy). Using multivariate logistic regression, the pretreatment ${ }^{99 \mathrm{~m}} \mathrm{Tc}$ MAA SPECT voxel value was the only significant factor associated with response by TLG, with a sensitivity of $89 \%$ and specificity of $65 \%$, positive predictive value of $71 \%$, and a negative predictive value of $87 \%$ [14].

Knešaurek et al. [26] reported a study to develop an objective measure of correlation between ${ }^{99 \mathrm{~m}} \mathrm{Tc}$-MAA SPECT pre treatment and ${ }^{90} \mathrm{Y}$ SPECT/CT Bremsstrahlung gamma emissions from the implanted resin microspheres [26]. In outlining the reasons why it is extremely difficult to utilize the very weak ${ }^{90} \mathrm{Y}$ gamma emissions from implanted microspheres, they pointed out that gamma emissions are part of a continuous energy spectrum, susceptible to scatter and septal penetration. Therefore, if ${ }^{99 \mathrm{~m}} \mathrm{Tc}$-MAA SPECT is to be used in treatment planning, it would be helpful to have an objective measure of reliability for the two activity distributions MAA and ${ }^{90} \mathrm{Y}$. These investigators concluded that Spearman's rank correlation value was superior to image distance for correlating the ${ }^{90} \mathrm{Y}$ microsphere and MAA activity distributions [26].

An alternate approach to the dosimetry dilemma in RE which does not rely on ${ }^{99 \mathrm{~m}}$ Tc-MAA activity distributions has been explored recently by Minarik et al. [48] These authors confirmed the feasibility of using quantitative ${ }^{90} \mathrm{Y}$ Bremsstrahlung SPECT imaging and Monte Carlo simulations in an experimental phantom and one clinical patient case. They concluded that although the ${ }^{90} \mathrm{Y}$ Bremsstrahlung spectrum is continuous with no pronounced peak and the count rate is low, an accurate activity estimate can be obtained with proper compensations applied to the reconstruction [48]. Lhommel et al. [49] imaged via PET scanner the actual ${ }^{90} \mathrm{Y}$ microsphere distribution in situ in a patient treated with resin microspheres for colorectal cancer liver metastases [49]. These authors assessed the biodistribution of ${ }^{90} \mathrm{Y}$ microspheres in situ by exploiting the internal pair production in ${ }^{90} \mathrm{Y}$ decay which occurs in 32 out of one million decays. The "time of flight" (TOF) regarding the detection of the $\mathbf{e}^{+} \mathbf{e}^{-}$with appropriate detectors and reconstruction software enabled accurate dose distribution estimates in a 3D dataset $[49,50]$. Although this approach is exciting and may prove helpful in the future, at present the availability of PET scanners that are capable of TOF is low and the image acquisition time is 40 minutes, which could be difficult for some patients to tolerate. Nevertheless, TOF data represent a potential breakthrough in solving dosimetry questions in ${ }^{90} \mathrm{Y}$ microsphere therapy. Werner et al. [50] used PET CT to assess the biodistribution of ${ }^{90} \mathrm{Y}$ microspheres without TOF. They determined that the minimum diameter of activity for detection was $17 \mathrm{~mm}$ [51]. 
Our research objective was to assess the feasibility of using ${ }^{99 \mathrm{~m}} \mathrm{Tc}$ MAA SPECT activity distributions prior to radioembolization as an aid to predicting toxicity and response by retrospectively applying an experimental software application. We noted some challenges to manual registration of SPECT and CT scans which could be more quickly accomplished with an automated process. In addition, there were a few situations in which patient position was different between scans and thus having a deformable registration option could be helpful. Higher resolution of the SPECT activity distribution is needed and this limits more accurate dose estimates. It is known from highly accurate human liver radiation three dimensional dose calculations $[12,25]$ using Monte Carlo processes that the 100 Gy isodose volume encompasses up to $4 \mathrm{~cm}$ in diameter region, however lower dose estimates were routinely seen in the present study.

Future improvements in RE dose estimation may include PET CT imaging of microspheres post treatment for more accurate dose estimates to tumor and normal liver. Another approach could include a treatment-planning gamma microsphere to replace the ${ }^{99 \mathrm{~m} T c-}$ MAA SPECT imaging, and the same microsphere with dual-labeling (therapeutic ${ }^{90} \mathrm{Y}$ plus diagnostic gamma isotope) could be used for confirmation of dose delivery.

\section{Conclusions}

Our study of converted ${ }^{99 m}$ Tc-MAA SPECT activity distributions into absorbed dose in liver and tumor provided reasonable estimates when reviewed from MIRD calculations and with clinical data. Response and toxicity findings were not significantly correlated with dose estimates. It is feasible to manually co-register ${ }^{99 \mathrm{~m}} \mathrm{Tc}-\mathrm{MAA}$ activity distributions with CT scan in a software module that uses Monte Carlo simulations to convert voxel values of normal liver and tumor into absorbed dose from a variety of isotopes, especially ${ }^{90} \mathrm{Y}$. The phantom measurements validated the Monte Carlo dose algorithm to within $2-3 \%$. The heterogeneity of the patient's tumors, prior treatment, and unpredictability of hepatic arterial flow at the time of treatment all impact response to radiation and microsphere distribution. This first step in developing a radiation treatment planning software tool was successful in identifying the many areas that will require further research and development to reach the goal of a clinically robust resource for radioembolization.

Disclosures by authors on potential conflict of interest: Andrew Kennedy, Consultant to Sirtex Medical, research grants and honoraria for providing CME lectures from Sirtex Medical. William Dezarn, Honoraria for providing CME lectures from Sirtex Medical. Alec Weiss, No potential conflicts to disclose.

\section{Acknowledgement}

The authors would like to thank the University of North Carolina at Chapel Hill and its Department of Radiation Oncology for providing the PLanUNC platform, and Mr. Andrew Kennedy, Jr for research assistance and data collection.

\section{References}

1. Kennedy A, Nag S, Salem R, Murthy R, McEwan AJ, et al. (2007) Recommendations for radioembolization of hepatic malignancies using yttrium-90 microsphere brachytherapy: a consensus panel report from the radioembolization brachytherapy oncology consortium. Int J Radiat Oncol Biol Phys 68: 13-23.

2. Kennedy AS, Salem R (2010) Radioembolization (yttrium-90 microspheres) for primary and metastatic hepatic malignancies. Cancer J 16: 163-175.

3. Kennedy AS (2009) Intraarterial Yttrium-90 Microspheres for Hepatic Malignancies, in ASCO Education Book. Am Soc Clin Oncol 251-255.

4. Lewandowski RJ, Sato KT, Atassi B, Ryu RK, Nemcek AA Jr, et al. (2007) Radioembolization with 90Y microspheres: angiographic and technical considerations. Cardiovasc Intervent Radiol 30: 571-592.
5. Salem R (2007) Radioembolization with $90 \mathrm{Y}$ microspheres: technical considerations. J Vasc Interv Radiol 18: 1460-1461.

6. Salem R, Thurston KG (2006) Radioembolization with yttrium-90 microspheres: a state-of-the-art brachytherapy treatment for primary and secondary live malignancies: part 3: comprehensive literature review and future direction. $J$ Vasc Interv Radiol 17: 1571-1593.

7. Salem R, Thurston KG (2006) Radioembolization with 90yttrium microspheres: a state-of-the-art brachytherapy treatment for primary and secondary liver malignancies. Part 2: special topics. J Vasc Interv Radiol 17: 1425-1439.

8. Salem R, Thurston KG (2006) Radioembolization with 90Yttrium microspheres: a state-of-the-art brachytherapy treatment for primary and secondary liver malignancies. Part 1: Technical and methodologic considerations. J Vasc Interv Radiol 17: 1251-1278.

9. Burton MA, Gray BN, Kelleher DK, Klemp PF (1990) Selective internal radiation therapy: validation of intraoperative dosimetry. Radiology 175: 253-255.

10. Burton MA, Gray BN, Klemp PF, Kelleher DK, Hardy N (1989) Selective internal radiation therapy: distribution of radiation in the liver. Eur $\mathrm{J}$ Cancer Clin Oncol 25: $1487-1491$

11. Campbell AM, Bailey IH, Burton MA (2000) Analysis of the distribution of intraarterial microspheres in human liver following hepatic yttrium-90 microsphere therapy. Phys Med Biol 45: 1023-1033.

12. Campbell AM, Bailey IH, Burton MA (2001) Tumor dosimetry in human live following hepatic yttrium-90 microsphere therapy. Phys Med Biol 46: 487-498.

13. Dhabuwala A, Lamerton P, Stubbs RS (2005) Relationship of 99mtechnetium labelled macroaggregated albumin (99mTc-MAA) uptake by colorectal live metastases to response following Selective Internal Radiation Therapy (SIRT) BMC Nucl Med 5: 7 .

14. Flamen P, Vanderlinden B, Delatte P, Ghanem G, Ameye L, et al. (2008) Multimodality imaging can predict the metabolic response of unresectable colorectal liver metastases to radioembolization therapy with Yttrium-90 labeled resin microspheres. Phys Med Biol 53: 6591-6603.

15. Fox RA, Klemp PF, Egan G, Mina LL, Burton MA, et al. (1991) Dose distribution following selective internal radiation therapy. Int J Radiat Oncol Biol Phys 21 463-467.

16. Gray BN, Burton MA, Kelleher D, Klemp P, Matz L (1990) Tolerance of the liver to the effects of Yttrium-90 radiation. Int J Radiat Oncol Biol Phys 18: 619-623.

17. Gulec SA, Mesoloras G, Dezarn WA, McNeillie P, Kennedy AS (2007) Safety and efficacy of Y-90 microsphere treatment in patients with primary and metastatic liver cancer: the tumor selectivity of the treatment as a function of tumor to liver flow ratio. J Transl Med 5: 15

18. Gulec SA, Mesoloras G, Stabin M (2006) Dosimetric techniques in 90Y-microsphere therapy of liver cancer: The MIRD equations for dose calculations. J Nucl Med 47: 1209-1211.

19. Ho S, Lau WY, Leung TW, Chan M, Chan KW, et al. (1997) Tumour-to-normal uptake ratio of $90 \mathrm{Y}$ microspheres in hepatic cancer assessed with $99 \mathrm{Tcm}$ macroaggregated albumin. Br J Radiol 70: 823-828.

20. Ho S, Lau WY, Leung TW, Chan M, Johnson PJ, et al. (1997) Clinica evaluation of the partition model for estimating radiation doses from yttrium-90 microspheres in the treatment of hepatic cancer. Eur J Nucl Med 24: 293-298.

21. Ho S, Lau WY, Leung TW, Chan M, Ngar YK, et al. (1996) Partition mode for estimating radiation doses from yttrium-90 microspheres in treating hepatic tumours. Eur J Nucl Med 23: 947-952.

22. Kennedy AS, Coldwell D, Nutting C, Murthy R, Wertman DE Jr, et al. (2006) Resin 90Y-microsphere brachytherapy for unresectable colorectal liver metastases: modern USA experience. Int J Radiat Oncol Biol Phys 65: 412425

23. Kennedy AS, Kleinstreuer C, Basciano CA, Dezarn WA (2010) Compute modeling of yttrium-90-microsphere transport in the hepatic arterial tree to improve clinical outcomes. Int J Radiat Oncol Biol Phys 76: 631-637.

24. Kennedy AS, McNeillie P, Dezarn WA, Nutting C, Sangro B, et al. (2009) Treatment parameters and outcome in 680 treatments of internal radiation with resin 90Y-microspheres for unresectable hepatic tumors. Int J Radiat Oncol Biol Phys 74: 1494-1500.

25. Kennedy AS, Nutting C, Coldwell D, Gaiser J, Drachenberg C (2004) Pathologic response and microdosimetry of (90)Y microspheres in man: review of four explanted whole livers. Int J Radiat Oncol Biol Phys 60: 1552-1563. 
Citation: Kennedy A, Dezarn W, Weiss A (2011) Patient Specific 3D Image-Based Radiation Dose Estimates for 90Y Microsphere Hepatic Radioembolization in Metastatic Tumors. J Nucl Med Radiat Ther 2:111. doi:10.4172/2155-9619.1000111

26. Knesaurek K, Machac J, Muzinic M, DaCosta M, Zhang Z, et al. (2010) Quantitative comparison of yttrium-90 (90Y)-microspheres and technetium99m (99mTc)-macroaggregated albumin SPECT images for planning 90Y therapy of liver cancer. Technol Cancer Res Treat 9: 253-262.

27. Reed GB Jr, Cox AJ Jr (1966) The human liver after radiation injury. Am J Pathol 48: 597-612.

28. Riaz A, Gates VL, Atassi B, Lewandowski RJ, Mulcahy MF, et al. (2010) Radiation Segmentectomy: A Novel Approach to Increase Safety and Efficacy of Radioembolization. Int J Radiat Oncol Biol Phys 79: 163-171.

29. Sangro B, Gil-Alzugaray B, Rodriguez J, Sola I, Martinez-Cuesta A, et al. (2008) Liver disease induced by radioembolization of liver tumors: description and possible risk factors. Cancer 112: 1538-1546.

30. Sarfaraz M (2001) Radiation dose distribution in patients treated with Y-90 Microspheres for non-resectable hepatic tumors. Int J Rad Biol Phys 51: 32-33.

31. Sarfaraz M, Kennedy AS, Cao ZJ, Sackett GD, Yu CX, et al. (2003) Physical aspects of yttrium-90 microsphere therapy for nonresectable hepatic tumors. Med Phys 30: 199-203.

32. Sarfaraz M, Kennedy AS, Lodge MA, Li XA, Wu X, et al. (2004) Radiation absorbed dose distribution in a patient treated with yttrium-90 microspheres for hepatocellular carcinoma. Med Phys 31: 2449-2453.

33. Young JY, Rhee TK, Atassi B, Gates VL, Kulik L, et al. (2007) Radiation dose limits and liver toxicities resulting from multiple yttrium-90 radioembolization treatments for hepatocellular carcinoma. J Vasc Interv Radiol 18: 1375-1382.

34. Lau WY, Kennedy AS, Kim YH, Lai HK, Lee RC, et al. (2010) A patient selection and activity planning guide for selective internal radiation therapy with yttrium-90 resin microspheres. Int J Radiat Oncol Biol Phys.

35. Toohey RE, Stabin MG, Watson EE (2000) The AAPM/RSNA physics tutoria for residents: internal radiation dosimetry: principles and applications. Radiographics 20: 533-546.

36. Kennedy AS, Salem R (2010) Radioembolization (Yttrium-90 Microspheres) for Primary and Metastatic Hepatic Malignancies. Cancer J 16: 163-175.

37. Erdi AK, Yorke ED, Loew MH, Erdi YE, Sarfaraz M, et al. (1998) Use of the fast Hartley transform for three-dimensional dose calculation in radionuclide therapy. Med Phys 25: 2226-2233.

38. Hao H, Bracewell RN (1987) A Three Dimensional DFT Algorithm Using the Fast Hartley Transform. PROCEEDINGS OF THE IEEE 75: 264-266.

39. Mainegra-Hing E, Rogers DW, Kawrakow I (2005) Calculation of photon energy deposition kernels and electron dose point kernels in water. Med Phys 32: 685699
40. (2004) Dosimetry of Beta Rays and Low-Energy Photons for Brachytherapy with Sealed Sources (Report 72). Journal of the ICRU 4.

41. Salem R, Lewandowski RJ, Gates VL, Nutting CW, Murthy R, et al. (2010) Research Reporting Standards for Radioembolization of Hepatic Malignancies. J Vasc Interv Radiol 22: 265-278.

42. Kennedy AS, Kleinstreuer C, Basciano CA, Dezarn WA (2009) Computer modeling of $90 \mathrm{y}$-microsphere transport in the hepatic arterial tree to improve clinical outcomes. Int J Radiat Biol Phys 76: 631-637.

43. Fajardo LF, Berthrong M (1988) Vascular lesions following radiation. Pathol Annu 1: 297-330.

44. Lawrence TS, Robertson JM, Anscher MS, Jirtle RL, Ensminger WD, et al. (1995) Hepatic toxicity resulting from cancer treatment. Int J Radiat Oncol Bio Phys 31: 1237-1248.

45. Lawrence TS, Ten Haken RK, Kessler ML, Robertson JM, Lyman JT, et al. (1992) The use of 3-D dose volume analysis to predict radiation hepatitis. Int $J$ Radiat Oncol Biol Phys 23: 781-788.

46. Pan CC, Kavanagh BD, Dawson LA, Li XA, Das SK, et al. (2010) Radiationassociated liver injury. Int J Radiat Oncol Biol Phys 76: S94-100.

47. Gulec SA, Sztejnberg ML, Siegel JA, Jevremovic T, Stabin M (2010) Hepatic structural dosimetry in $(90) \mathrm{Y}$ microsphere treatment: a Monte Carlo modeling approach based on lobular microanatomy. J Nucl Med 51: 301-310.

48. Minarik D, Sjogreen Gleisner K, Ljungberg M (2008) Evaluation of quantitative (90)Y SPECT based on experimental phantom studies. Phys Med Biol 53 5689-5703.

49. Lhommel R, Goffette P, Van den Eynde M, Jamar F, Pauwels S, et al. (2009) Yttrium-90 TOF PET scan demonstrates high-resolution biodistribution after liver SIRT. Eur J Nucl Med Mol Imaging 36: 1696.

50. Lhommel R, van Elmbt L, Goffette P, Van den Eynde M, Jamar F, et al. (2010) Feasibility of (90)Y TOF PET-based dosimetry in liver metastasis therapy using SIR-Spheres. Eur J Nucl Med Mol Imaging 37: 1654-1662.

51. Werner MK, Brechtel K, Beyer T, Dittmann H, Pfannenberg C, et al. (2010) $\mathrm{PET} / \mathrm{CT}$ for the assessment and quantification of (90)Y biodistribution after selective internal radiotherapy (SIRT) of liver metastases. Eur J Nucl Med Mol Imaging 37: 407-408

\section{Footnote}

1. PLanUNC: Sha $X$ Chang $\mathrm{PhD}$ : Head Physics \& Computing Division, Department of Radiation Oncology, University of North Carolina School of Medicine, Campus Box 7512, Chapel Hill, NC 27599-7512

2. http://www.fda.gov/cder/cancer/toxicityframe.htm 